\title{
TEMPERAMENTO E SUA RELAÇÃO COM PROBLEMAS EMOCIONAIS E DE COMPORTAMENTO EM PRÉ-ESCOLARES'1
}

\author{
Sofia Muniz Alves Gracioli² \\ Maria Beatriz Martins Linhares \\ Universidade de São Paulo, Ribeirão Preto-SP, Brasil
}

\begin{abstract}
RESUMO. O temperamento é uma variável da pessoa que interage com variáveis ambientais e pode influenciar trajetórias de desenvolvimento de crianças. Estudos sobre o desenvolvimento emocional e social têm destacado a importância do temperamento como uma variável que influencia fundamentalmente o ajustamento saudável do indivíduo. O presente estudo teve por objetivo revisar de forma sistemática estudos empíricos, publicados de 2007 a 2012, sobre o tema temperamento e suas relações com problemas emocionais e de comportamento em crianças na fase pré-escolar. Foram selecionados sistematicamente dezoito estudos empíricos indexados nas bases de dados PubMed, PsycINFO e Scielo. Os resultados dos estudos evidenciaram, predominantemente, relações diretas entre fatores do temperamento e os tipos específicos de problemas emocionais e de comportamento internalizantes e externalizantes, em crianças na fase pré-escolar. O temperamento com traços de retraimento, timidez e medo mostrou associação com problemas internalizantes, enquanto o temperamento do tipo difícil ou com falta de controle inibitório comportamental mostrou associação com problemas externalizantes. Além disso, verificou-se que variáveis da criança do tipo nascimento prematuro, transtorno do autismo e alto nível de cortisol salivar, assim como variáveis de risco psicossocial do tipo baixo nível socioeconômico e depressão materna, foram fatores moderadores das relações entre os traços de temperamento e problemas de comportamento.
\end{abstract}

Palavras-chave: Temperamento; problemas de comportamento; pré-escolares.

\section{TEMPERAMENT RELATED TO EMOTIONAL AND BEHAVIORAL PROBLEMS IN PRESCHOOL}

\begin{abstract}
Temperament is a variable of the person who interacts with environmental variables, and can influence children's developmental trajectories. Studies on emotional and social development have highlighted the relevance of temperament as a variable that fundamentally influence the adjustment healthy individual. The aim of the present study was to systematically review empirical studies, published from 2007 to 2012, on the temperament and its relationship with emotional and behavioral problems in children in pre-school age. We selected 18 empirical studies indexed in PubMed, PsycINFO and Scielo data. The results showed, predominantly, direct associations between temperament factors and specific types of emotional and internalized and externalized behavior problems, in pre-school children. The temperament with withdrawal symptoms, shyness and fear traits were associated with internalized problems. Otherwise, the temperament with dificulty trait and without behavior innhibitory control showed associations with externalized problems. In addition, child variables, as pre-term birth, autism disorder, and high level of salivary cortisol, and psychosocial risk variables, as low socioeconomic level and maternal depression, were moderator factors of the relationships between temperament traits and behavior problems.
\end{abstract}

Key words: Temperament; behavioral problems; preschoolers.

\section{EL TEMPERAMENTO Y SU RELACIÓN CON LOS PROBLEMAS EMOCIONALES Y DE COMPORTAMIENTO EN EL PREESCOLAR}

RESUMEN. El temperamento es una variable de la persona que interactúa con variables ambientales y puede influir en las trayectorias de desarrollo de los niños. Estudios sobre el desarrollo emocional y social han puesto de relieve la importancia del temperamento como una variable que influye principalmente en el ajuste sano del

\footnotetext{
Apoio e financiamento: Coordenação de Aperfeiçoamento de Pessoal de Nível Superior (CAPES).

2 Endereço para correspondência: Avenida 10, 1245, centro, Orlândia-SP, CEP 14.620 000. E-mail: sofiagracioli@yahoo.com.br
} 
individuo. Este estudio tuvo como objetivo realizar una revisión sistemática de estudios empíricos publicados de 2007 a 2012, sobre el tema de temperamento y de sus relaciones con los problemas emocionales y de comportamiento en los niños en edad preescolar. Fueron seleccionados sistemáticamente 18 estudios empíricos indizados en las bases de datos PubMed, PsycINFO y SciELO. Los resultados de los estudios mostraron, predominantemente, relaciones directas entre factores de temperamento y tipos específicos de problemas emocionales y de comportamientos de internalización y externalización, en niños en edad preescolar. El temperamento con rasgos de retraimiento, timidez y miedo señaló asociación con problemas de internalización, mientras que el temperamento del tipo difícil o con falta de control inhibitorio de comportamiento señaló asociación con problemas de externalización. Además, se verificó que las variables del niño, como nacimiento prematuro; trastorno del autismo y alto nivel de cortisol salival, así como las variables de riesgo psicosocial, del bajo nivel socioeconómico y depresión materna fueron factores moderadores de las relaciones entre los rasgos de temperamento y problemas de comportamiento.

Palabras clave: Temperamento; problemas de comportamiento; preescolares.

O temperamento surge da herança genética e pode ser influenciado pelas experiências, integrando a personalidade do indivíduo (Rothbart, Ahadi \& Evans, 2000; Rothbart \& Putnam, 2002). Trata-se de um conjunto de diferenças individuais estáveis de forte base genética e neurobiológica que aparecem a partir do nascimento. As diferenças individuais do temperamento expressam a constituição mais precoce da personalidade e o substrato mais preservado evolutivamente no desenvolvimento. Estudos sobre o desenvolvimento emocional e social têm destacado a importância do temperamento como uma variável que influi fundamentalmente no ajustamento saudável do indivíduo (Prior et al., 2008).

O estudo do temperamento compreende três principais abordagens teórico-metodológicas: a abordagem de Estilo Comportamental, de Thomas e Chess (1977), a abordagem de Bus e Plomin (1984) e a abordagem de Rothbart (1981).

A primeira delas foi pioneira em avaliar 0 temperamento, focalizando a maneira pela qual este se manifesta, sua razão e suas características observáveis por meio do comportamento (Muris \& Ollendick, 2005). Nesta abordagem, a classificação proposta dos tipos de temperamento inclui: o temperamento fácil, caracterizado por regularidade nas funções biológicas, respostas de aproximação positiva a estímulos novos; o temperamento difícil, caracterizado por sinais de irregularidade nas funções biológicas, respostas de retraimento negativo a novos estímulos e desadaptação ao ambiente; e o temperamento lento para reagir, caracterizado pela combinação de respostas negativas a estímulos novos com adaptabilidade lenta após contatos repetidos.
Na segunda abordagem, proposta por Buss e Plomin (1984), o temperamento é definido como um conjunto de traços de personalidade hereditários que aparecem cedo no desenvolvimento, sendo constituído por três traços específicos, a saber: emocionalidade, atividade e sociabilidade (EAS).

$\mathrm{Na}$ terceira abordagem, 0 modelo psicobiológico desenvolvido por Rothbart (1981), o temperamento é entendido como diferenças individuais com base constitucional na reatividade e autorregulação, influenciadas ao longo do tempo pela hereditariedade, maturação e experiência. De acordo com a abordagem de Rothbart, o temperamento tem três fatores, a saber: Afeto negativo, Extroversão e Controle com esforço. Cada fator reúne as respectivas dimensões, a saber: frustração, medo, tristeza, capacidade de se acalmar, desconforto e sensibilidade perceptual (Afeto negativo); antecipação positiva, nível de atividade e impulsividade (Extroversão); e prazer de baixa intensidade, controle inibitório e focalização da atenção (Controle com esforço).

O Controle com esforço do temperamento entra como um importante fator para 0 desenvolvimento da autorregulação (Rothbart \& Bates, 2006; Feldman, 2009; Sameroff, 2009). Ao longo do desenvolvimento as crianças sofrem influências dos processos de regulação. $\mathrm{Na}$ fase neonatal aparecem: a regulação fisiológica, em que ocorrem a modulação do estado de alerta fisiológico, a capacidade de se acalmar e a resposta do cortisol a estressores; a regulação emocional, que permite ajustamento das respostas afetivas, de atenção e de comportamento motor voluntário de forma dirigida ao alcance dos objetivos; a regulação da atenção, que acontece na fase pré-escolar, incluindo os mecanismos da atenção do 
pensamento simbólico, memória e automonitoramento do comportamento; a regulação do comportamento, que permite 0 ajuste do comportamento de acordo com as demandas sociais (Olson, Sameroff, Lunkenheimer \& Kerr, 2009). Os processos de autorregulação, que envolvem a capacidade de monitorar o comportamento em resposta a diferentes demandas situacionais, permitem uma melhor compreensão dos processos envolvidos no interjogo entre fatores de risco e mecanismos de proteção nas trajetórias de desenvolvimento do indivíduo, especialmente na fase do desenvolvimento pré-escolar. Nesta fase a criança aprende a adaptar-se flexivelmente às situações que exigem diferentes padrões de conduta.

Neste contexto, torna-se evidente a necessidade de compreender as relações entre temperamento da criança e comportamento. 0 presente estudo teve por objetivo analisar a literatura indexada e atualizada, publicada nos últimos cinco anos, a fim de verificar se há relação entre temperamento e problemas emocionais e de comportamento em crianças na fase pré-escolar.

\section{MÉTODO}

A presente revisão bibliográfica foi realizada por meio da seleção de artigos científicos nas bases de dados PubMed, PsycINFO, Scielo. As palavras-chave selecionadas para a revisão bibliográfica foram: Temperament and Behavior e Temperament and Emotional. No PubMed foram utilizados os seguintes limites: humans; all infant birth-23 months; newborn birth-1 month; infant 1-23 months; preschool child 2-5 years.

Os critérios de inclusão foram os seguintes: os artigos terem sido publicados nos últimos cinco anos; o estudo ser empírico, avaliando temperamento, comportamento e problemas emocionais; o estudo estabelecer a relação entre temperamento e comportamento; a amostra ser constituída de crianças na fase pré-escolar; o artigo ter sido publicado nos idiomas Português, Inglês ou Espanhol. Os critérios de exclusão, por sua vez, foram: artigos de revisão, comentários, editoriais ou cartas; estudos que não avaliaram como variáveis principais o temperamento, o comportamento e problemas emocionais; estudos que avaliaram outras faixas etárias diferentes da idade-alvo, que é a fase pré- escolar; e artigos em outros idiomas que não em Português, Inglês ou Espanhol.

$\mathrm{Na}$ busca inicial foram encontrados 357 artigos nas bases de dados (223 no PubMed, 134 no PsycINFO, nenhum no Scielo e nenhum no Lilacs). Em primeiro lugar foram retirados os artigos repetidos, e em segundo lugar, após a leitura dos resumos, foram aplicados os critérios de inclusão e exclusão. Dos 339 artigos, foram eliminados 321, a saber: estudos cujo objetivo principal não fosse o de analisar a relação entre temperamento e problemas emocionais e/ou de comportamento das crianças $(n=109)$; estudos com faixas etárias das crianças diferentes da fase pré-escolar $(n=98)$; artigos de revisão $(n=26)$; estudos em outros idiomas $(n=30)$; estudos psicométricos $(n=31)$; e artigos publicados antes de 2007 ( $n=27)$. A amostra final analisada no presente estudo foi de dezoito artigos.

\section{RESULTADOS}

\section{Características dos estudos}

Os dezoito artigos revisados foram publicados predominantemente no ano de 2009 $(n=7 ; 38 \%)$, e os demais, distribuídos em 2008 $(n=3 ; 16 \%), 2010 \quad(n=4 ; 23 \%)$ e $2011 \quad(n=4$; 23\%). Com relação ao delineamento das pesquisas, foram onze estudos longitudinais $(62 \%)$ e sete estudos de corte transversal. As amostras de crianças dos estudos eram procedentes predominantemente da América do Norte $(n=11$ estudos, sendo nove dos EUA e dois do Canadá), seguidos da Europa $(n=4$ estudos; sendo um de Amsterdã, na Holanda, um da Inglaterra, um da Suécia e um da Itália), além da Oceania (Austrália, com dois estudos), e da Ásia (China, com um estudo).

Quanto aos instrumentos para avaliar o temperamento das crianças, observou-se grande variedade de instrumentos e medidas, incluindo onze instrumentos diferentes (The Infant Behavior Questionnaire - IBQ, Carey Infant Temperament Scale - CTS, Toddler Behavior Assessment Questionnaire - TBAQ, The Emotionality, Activity, Sociability, and Impulsivity - EASI, Infant Characteristic Questionnaire - ICQ, Short Infant and Toddler Temperament Questionnaires -STSC, Infant Behavior Record IBR, Questionário Italiano do Temperamento QUIT, The Children's Behavior Questionnaire CBQ e Chinese Toddler Temperament Scale). O 
instrumento mais utilizado nas avaliações foi o Infant Behavior Questionnaire - IBQ $(n=17)$, desenvolvido por Rothbart (1981).

Dos dezoito estudos, $90 \%$ utilizaram escalas de avaliação do temperamento e comportamento, e no restante $(10 \%)$ foi utilizada a metodologia de observação sistemática de indicadores de temperamento e comportamento das crianças. Foram utilizados seis tipos de instrumento: Child Behavior Checklist - CBCL, Scale related to externalizing and internalizing problems, Temperament Questionnaire in Italian - QUIT, Communication and Symbolic Behavior Scales - Developmental Profile - CSBS-DP e Behavior Assessment Battery - BCL, sendo predominantemente utilizado o Child Behavior Checklist - CBCL (Achenbach \& Rescorla, 2000).

\section{Principais achados dos estudos}

Os resultados dos artigos da presente revisão foram organizados de acordo com o delineamento, a saber: os estudos de comparação entre grupos e os estudos de análise de predição.

Estudos de comparação entre grupos, de acordo com o tipo de temperamento das crianças

Crianças avaliadas com temperamento típico entre 24 a 48 meses apresentaram menos problemas de comportamento, tanto do tipo internalizante quanto do externalizante, quando comparadas a crianças com temperamento expressivo e com medo. As crianças com temperamento do tipo medo, por sua vez, apresentaram maiores escores de problemas de comportamento internalizantes, quando comparadas às crianças com temperamento do tipo expressivo. Estas apresentaram mais problemas de comportamento externalizantes do que as crianças com perfil de temperamento com medo (Akker, Dekovic, Prinzie \& Asscher, 2010).

O efeito dos temperamentos dos tipos fácil e difícil, em associação ao risco do contexto ambiental, foi examinado no comportamento de crianças aos 36 meses. O temperamento difícil era caracterizado por serem as crianças menos sintonizadas com as demandas ambientais, terem maior risco para apresentar dificuldades comportamentais e desenvolvimento de psicopatologias (Derauf et al., 2011). As crianças com temperamento fácil (mais adaptadas socialmente e com comportamentos positivos), quando estavam inseridas em um ambiente de baixo risco quanto ao nível socioeconômico, apresentaram menos problemas de comportamento, tanto internalizantes quanto externalizantes; mas quando havia alto risco ambiental, mesmo as crianças que apresentavam temperamento do tipo fácil apresentaram problemas de comportamento. Notou-se neste estudo forte relação entre o temperamento da criança e o contexto ambiental familiar em que ela estava inserida.

Uma amostra de crianças de 24 meses foi distribuída em grupos, de acordo com características do temperamento, em exuberante, inibido e pouco reativo (Stifter, Putnam \& Jahromi, 2008). O temperamento exuberante apresentava características como emocionalidade positiva e sociabilização. Verificou-se que, mesmo com a presença dessas características positivas, ao serem comparadas com as crianças com temperamento inibido ou pouco reativo, essas crianças de temperamento exuberante apresentaram menos problemas de comportamento internalizantes e externalizantes. Um fator moderador para a diminuição de problemas de comportamento foi a regulação emocional por parte dos pais, cuidadores e professores, os quais estabeleciam regras de conduta e eliminavam os comportamentos indesejados das crianças.

Estudos de comparação entre grupos, de acordo com transtornos do desenvolvimento

Dois estudos avaliaram crianças com transtorno de desenvolvimento como autismo, síndromes e atrasos no desenvolvimento, comparadas a crianças com desenvolvimento típico. O temperamento foi avaliado em três grupos de crianças de 24 meses, a saber: crianças autistas com irmãos autistas, crianças autistas que não tinham irmãos autistas e um grupo-controle (Garon et al., 2009). O temperamento das crianças autistas com irmãos autistas apresentou alto afeto negativo, maior propensão a ter medo, ansiedade, tristeza e raiva, quando comparadas às dos outros dois grupos. Quanto à avaliação do comportamento, as crianças com irmãos autistas apresentaram pobre controle da atenção, não respondendo bem às pistas observadas (capacidade de guiar e manter o foco de atenção sobre determinado estímulo), além de apresentarem menor sensibilidade perceptual, quando comparadas às 
dos outros dois grupos. O temperamento com alto traço de afeto negativo provocou nas crianças problemas relacionados ao controle da atenção e do comportamento, apresentando problemas externalizantes.

O temperamento de crianças com transtornos mistos (síndromes, autismo e atraso no desenvolvimento) foi avaliado aos 36 meses e comparado a um grupo-controle de crianças com desenvolvimento típico (Bostrom, Broberg \& Hwang, 2010). As crianças do grupo com transtornos mistos apresentaram temperamento mais tímido e com impulsividade, além de menor nível de atividade (menor energia) do que 0 grupo-controle. Além disso, as crianças com transtornos mistos apresentaram menos sociabilidade, preferindo ficar sozinhas a buscarem a companhia de outras pessoas.

\section{Estudos de comparação entre grupos, de acordo com o nascimento prematuro}

Crianças nascidas pré-termo (<37 semanas de idade gestacional) apresentaram menor adaptação social entre 18 e 36 meses, quando comparadas com as crianças nascidas a termo ( $\geq 37$ semanas de idade gestacional) (Hwang, Soong \& Liao, 2009); mas crianças prematuras com temperamento fácil apresentaram melhor adaptação social. Por outro lado, crianças nascidas pré-termo apresentaram, aos cinco anos, melhor capacidade de expressar as emoções positivas, sendo mais sociáveis e buscando a companhia de outras pessoas, do que as crianças nascidas a termo (Perricone \& Morales, 2011); no entanto, crianças prematuras apresentaram problemas de diminuição da atenção e pior controle motor. Notou-se que a alta reatividade associada ao nascimento prematuro desencadeou problemas de comportamento.

\section{Estudos de predição sobre temperamento, problemas de comportamento e psicopatologias}

O temperamento difícil de crianças nascidas pré-termo com muito baixo peso, avaliado aos 12 meses, associado à presença de conflitos familiares vivenciados aos seis anos, foi preditor de problemas de comportamento externalizante aos oito anos de idade (Whiteside-Mansell, Bradley, Casey, Fussell \& Conners-Burrow, 2009).

O temperamento das crianças caracterizado como de baixo controle inibitório e alto nível de raiva e impulsividade, aos nove meses, foi preditor de problemas de comportamento externalizantes aos quatro anos de idade. Por outro lado, quando as crianças apresentavam baixos níveis de impulsividade, de atenção e de baixo controle inibitório, ocorriam problemas internalizantes aos quatro anos (Eisenberg et al., 2009).

O temperamento com afeto negativo avaliado aos nove meses, associado ao hormônio do estresse (cortisol) medido aos quatro anos, foi preditor de problemas de comportamento retraído, especialmente em meninos desta mesma idade (quatro anos) (Edgar et al., 2008). As crianças com temperamento tímido apresentaram deficiências quanto ao desenvolvimento da comunicação social aos 24 meses. As características do temperamento das crianças, combinadas com variáveis maternas (mães insatisfeitas com sua vida conjugal e depressivas) afetaram a competência comunicativa das crianças, especialmente em vocabulário e simbolismo (Prior et al., 2008).

Crianças gêmeas, avaliadas entre 12 e 36 meses, apresentaram temperamento com baixo controle inibitório e maior nível de raiva. $\mathrm{O}$ baixo controle inibitório foi preditor de diagnóstico de transtorno de ansiedade (ansiedade generalizada e fobia social) aos seis anos de idade, transtorno que se caracteriza por ser um problema de comportamento internalizante (Gagne \& Goldsmith, 2011).

Crianças avaliadas aos quatro anos apresentarem temperamento com alta inibição comportamental (estilo de temperamento caracterizado pela cautela e prevenção em situações sociais) foi preditor do diagnóstico de ansiedade, fobia social e ansiedade generalizada também aos seis anos de idade (Hudson, Dodd, Lyneham \& Bovopoulous, 2009). As mães superprotetoras que também apresentaram ansiedade potencializavam 0 diagnóstico de ansiedade dos filhos, porquanto a condição de superproteção materna tirava a autonomia das crianças.

A depressão materna e paterna foi avaliada na fase de seis a oito meses dos bebês e verificou-se a relação entre temperamento e comportamento das crianças. A depressão materna foi preditora do temperamento difícil das crianças aos 24 meses, com dificuldades de humor e menor intensidade de energia; mas notou-se que houve um efeito diferencial do 
gênero das crianças. Quando a mãe apresentava depressão, o temperamento - tanto dos meninos quanto das meninas - era afetado; por outro lado, quando a depressão era paterna, o efeito negativo no temperamento foi observado apenas nos meninos (Hanington, Ramchandani \& Stein, 2010).

Quatro estudos não evidenciaram associação significativa entre temperamento e comportamento. Ao verificarem a influência de uma alimentação calórica durante a noite como possibilidade de aumento do risco de obesidade aos três anos, o comportamento não se mostrou associado a características do temperamento das crianças (Vollrath, Tonstad, Rothbart \& Hampson, 2011).

O comportamento antissocial da criança e a depressão materna, avaliados aos cinco meses, foram preditores do comportamento de desrespeitar regras, entre 29 e 74 meses de idade. Porém, ao verificar a influência do temperamento difícil, este não foi preditor de problemas de comportamento (Petitclerc, Boivin, Dionne, Zoccolillo \& Tremblay, 2009).

$\mathrm{Na}$ avaliação do temperamento e comportamento de crianças, foi verificado que, quando o pai não residia na mesma casa do que os filhos, as crianças apresentavam problemas de comportamento e temperamento somente quando este também tinha problemas de comportamento (Flouri \& Malmberg, 2010).

\section{DISCUSSÃO}

A presente revisão sistemática da literatura constatou que traços do temperamento estão diretamente relacionados com problemas de comportamento e emocionais da criança. A fase pré-escolar permite verificar a relação direta entre temperamento e comportamento, por meio dos processos de regulação da atenção e comportamento das crianças (Olson et al., 2009).

Com relação à verificação de quais traços do temperamento estão diretamente ligados aos tipos específicos de problemas de comportamento, internalizantes ou externalizantes, a dimensão elevada do temperamento afeto negativo (frustração, medo, tristeza, capacidade de se acalmar, desconforto e sensibilidade perceptual) foi preditora de mais problemas de comportamento do tipo internalizante (Edgar et al., 2008). Outro fator do temperamento, o baixo controle com esforço (prazer de baixa intensidade, controle inibitório e focalização da atenção), foi preditor de mais problemas de comportamento do tipo externalizante (Prior et al., 2008). Comportamentos marcados por hiperatividade, impulsividade, oposição, agressão, desafio e manifestações antissociais são classificados como externalizantes, em oposição a padrões de comportamento internalizantes que incluem: disforia, retraimento, medo e ansiedade. Os problemas externalizantes tendem a ser mais estáveis do que os internalizantes e têm curso e prognóstico menos favoráveis, particularmente os componentes de agressividade, impulsividade e tendências antissociais esses representam as formas mais comuns e persistentes de desajustamento na meninice e são precursores de distúrbio de conduta na adolescência (Esser, Schmidt \& Woerner 1990). Tais comportamentos associados a ajustamento social pobre têm consequências crônicas e graves não apenas para as crianças que os manifestam, mas também para os pais, irmãos, professores e a sociedade em geral. Essas crianças estão em risco de rejeição pelos companheiros, conflitos com a família e com os professores, fracasso escolar, dificuldades ocupacionais, além do risco mais sério para comportamentos socialmente desviantes (Olson et al., 2009).

O temperamento apresenta várias abordagens para sua classificação, as principais são três abordagens de: Tomas e Chess (1977), Buss e Plomim (1984) e Rothbart (1981). Na presente revisão verificou-se que houve maior porcentagem de estudos com a abordagem de Rothbart, o temperamento avaliado por três fatores: afeto negativo, extroversão e controle com esforço. O fator controle com esforço permite o controle voluntário do comportamento e da emoção, sendo o fator do temperamento que controla o impacto negativo dos outros fatores. Esse fator exerce uma forte influência no comportamento, particularmente nos últimos anos da infância, na adolescência e na vida adulta (Rothbart, 2004). Na fase pré-escolar pode-se melhor apreciar a interrelação entre os três fatores do temperamento, na medida em que se espera que o controle com esforço esteja mais bem organizado do que em etapas anteriores do desenvolvimento. Além disso, se espera que atue em conjunto com outros fatores moderando os traços do afeto negativo e a extroversão. 
As crianças com 0 temperamento caracterizado com humor negativo, medo, timidez e raiva mostram-se suscetíveis a apresentar problemas de desenvolvimento. As crianças com habilidades relacionadas ao controle com esforço, que indica capacidade de regulação fisiológica e comportamental, controlando e inibindo um comportamento dominante a favor de um comportamento subdominante, obtiveram resultados desenvolvimentais positivos (Watamura, Donzella, Kertes \& Gunnar, 2004).

Os achados da presente revisão mostraram que o temperamento difícil, crianças que apresentaram dificuldade de buscar a companhia de outras crianças, além de terem maior dificuldade a respeitar limites e regras, foi diretamente associado à possibilidade de desencadeamento de problemas de sociabilização (Akker et al., 2010; Edgar et al., 2008; Whiteside-Mansell et al., 2009). A emocionalidade negativa, por sua vez, em que as crianças apresentaram dificuldade de expressar suas emoções, foi associada a problemas do comportamento dos tipos internalizante e externalizante (Derauf et al., 2011; Hanington et al., 2010; Gagne \& Goldsmith, 2011; Eisenberg et al., 2009).

Ao analisar a relação temperamento e comportamento, foram verificados possíveis efeitos das variáveis biológicas, do tipo cortisol, nascimento prematuro (Edgar et al., 2008; Whiteside-Mansell et al., 2009), assim como variáveis ambientais do tipo risco psicossocial ambiental, superproteção materna, conflito familiar e depressão dos pais (Prior et al., 2008; Whiteside-Mansell et al., 2009; Hudson., 2009). O cortisol é um hormônio intimamente ligado ao sistema emocional, que serve para controlar inflamações, alergias, os níveis de estresse, diminuir a imunidade, manter a estabilidade emocional, estimular o açúcar do sangue e criar proteínas, o cortisol e o peso ao nascimento. $O$ cortisol alto, assim como o menor peso ao nascimento são preditores de maiores problemas com relação ao desenvolvimento das crianças. Essas variáveis mostraram efeitos que aumentaram 0 impacto na relação entre temperamento e comportamento das crianças (Prior et al., 2008; Edgar et al., 2008; Hudson et al., 2009).

As variáveis relacionadas ao ambiente no qual a criança está inserida (os riscos psicossociais e os conflitos familiares), associadas aos cuidados e às condições parentais (superproteção materna e depressão), foram preditoras da ocorrência de maiores problemas no desenvolvimento destas crianças. Em relação aos cuidados maternos, observa-se a importância destes nos primeiros anos de vida da criança, na medida em que os bebês dependem fortemente de seus cuidadores para auxiliar na regulação da emoção (Eisenbergetal., 2009). Neste sentido, torna-se importante a integridade emocional materna e aspectos da sua personalidade. A capacidade autorregulatória das crianças é fortemente influenciada pela experiência de regulação oferecida pelos cuidadores das crianças (Feldman, 2009; Sameroff, 2009).

Variáveis familiares podem contribuir para a persistência dos problemas da fase pré-escolar à escolar (Denham et al., 2000). Pesquisas recentes, ao avaliarem adolescentes de grupos antissociais, observaram que o desenvolvimento desse comportamento começa no lar durante a meninice. As práticas educativas coercitivas e punitivas contribuem para esta cadeia de adversidades, que por sua vez influenciam o desenvolvimento de agressão e fracasso escolar, levando à seleção de companheiros antissociais (Collins, Maccoby, Steinberg, Hetherington \& Bornstein, 2000).

A análise dos critérios de composição das amostras dos estudos revelou que a maioria destes selecionou crianças previamente identificadas com transtornos de desenvolvimento ou condições de risco ao nascimento (prematuridade, baixo peso). $\mathrm{Na}$ presente revisão houve uma predominância de estudos com crianças com desenvolvimento atípico autistas (Garon et al., 2009) e com transtornos mistos de desenvolvimento (atraso mental, síndromes).

Com relação à análise dos procedimentos utilizados para avaliação do temperamento e comportamento, foi predominante 0 uso de questionários, em sua maioria, respondidos pelas mães. O relato dos pais para avaliação do temperamento tem como vantagem o fato destes verem seus filhos em uma ampla variedade de situações, ética e logisticamente impossíveis de serem recriadas em situação artificial de laboratório (Rothbart, 2004).

Os questionários para avaliação de temperamento em crianças, baseados no relato dos pais, tem suas limitações. A personalidade dos pais e o modo como estes interpretam 0 
comportamento das crianças podem levar a vieses na obtenção de informações. Os pais inconscientemente fazem comparações ao julgar as características do seu filho, sujeitas às influências de experiências anteriores, não relacionadas com seu temperamento. Segundo Rothbart e Goldsmith (1981) essas preocupações podem ser sanadas com 0 cuidado na construção e apresentação dos itens dos questionários, perguntando apenas sobre eventos que ocorreram recentemente e sobre comportamentos infantis concretos, em vez de pedir aos pais para fazerem resumos ou julgamentos comparativos. Os questionários de Rothbart incluem avaliações por meio de heterorrelato dos cuidadores principais das crianças de três meses até 10 anos, ou autorrelato, para as faixas etárias de nove anos até a idade adulta (Evans \& Rothbart, 2007).

De acordo com Rothbart e Bates (2006), o relato dos pais apresenta grandes vantagens, pois estes são capazes de observar os seus filhos em várias situações e em diferentes momentos do dia, além de poderem estabelecer um grau razoável de validade objetiva. Por outro lado, as observações em laboratório permitem aos pesquisadores o controle do ambiente, possibilitando comparar o comportamento das crianças em situações semelhantes. Desta forma reduz consideravelmente as potenciais influências externas sobre o comportamento da criança. No entanto, há restrições na medida em que precisa ser escolhida uma dimensão do temperamento como foco da situação observada. Tanto os questionários, quanto as observações sistemáticas, são métodos válidos para avaliação do temperamento de crianças e sua escolha depende do objetivo da avaliação e da dimensão específica a ser avaliada.

A maioria dos estudos da presente revisão foram longitudinais, com análise de predição, possibilitando o exame de múltiplas variáveis no decorrer do desenvolvimento das crianças. O temperamento e comportamento foram analisados interligando complexas interrelações com outras variáveis da criança, tais como peso ao nascimento e cortisol, e com variáveis do ambiente, tais como os conflitos familiares. Os estudos longitudinais são de grande importância para verificação de inúmeras variáveis em períodos diferentes no transtorno do desenvolvimento, tendo relevância também para futuros estudos focalizados em prevenção de problemas no desenvolvimento.
Deve-se salientar que em dois dos estudos houve uma limitação relacionada ao uso de um mesmo instrumento para avaliar tanto o temperamento quanto o comportamento (Vollrath et al., 2011; Perricone et al., 2011). Um dos estudos utilizou o instrumento do comportamento QUIT (Questionário Italiano do Temperamento) para avaliar tanto temperamento quanto comportamento (Perricone et al., 2011). Em outro estudo que avaliou a obesidade e sua relação com temperamento e comportamento o instrumento do temperamento $\mathrm{CBQ}$ (The Children's Behavior Questionnaire) foi utilizado para avaliar apenas o comportamento das crianças (Vollrath et al., 2011). Esta é uma limitação importante, pois o instrumento $\mathrm{CBQ}$ é um instrumento validado para avaliação do temperamento e não para comportamentos.

\section{CONCLUSÃO}

A presente revisão evidenciou, predominantemente, relações diretas entre fatores do temperamento e os tipos específicos de problemas emocionais e de comportamento internalizantes e externalizantes de crianças na fase pré-escolar. O temperamento com traços de retraimento, timidez e medo mostrou associação com problemas internalizantes, enquanto 0 temperamento do tipo difícil ou com falta de controle inibitório comportamental mostrou associação com problemas externalizantes. Além disso, verificou-se que variáveis da criança dos tipos nascimento prematuro, transtorno do autismo e nível de cortisol salivar, assim como variáveis de risco psicossocial, dos tipos baixo nível socioeconômico e depressão materna, foram fatores moderadores da relação entre os traços de temperamento e problemas de comportamento das crianças. As pesquisas mostraram avanços na análise de múltiplas variáveis associadas ao temperamento, por meio de estudos longitudinais e análises de predição.

\section{REFERÊNCIAS}

Akker, A. L., Dekovic, M., Prinzie, P., \& Asscher, J. J. (2010). Toddlers' temperament profiles: stability and relations to negative and positive parenting. Journal of Abnormal Child Psychology, 38(4), 485495.

Bostrom, P., Broberg, M., \& Hwang, C. P. (2010). Different, difficult or distinct? Mothers' and fathers' perceptions of temperament in children with and 
without intellectual disabilities. Journal of Intellectual Disability Research, 54(9), 806-819

Buss, A. H. \& Plomin, R. (1984) Temperament: Early developing personality traits. Hillsdale, $\mathrm{NJ}$ : Lawarence Erbaum Associates.

Collins, W. A., Maccoby, E. E., Steinberg, L., Hetherington, E. M., \& Bornstein, M. H. (2000). Contemporary Research on Parenting: The Case for Nature and Nurture. American Psychologist, 55(2), 218-232.

Denham, S. A., Workman, E., Cole, P., Weissbrod, C., Kendziora, K., \& Zahn-Waxler, C. (2000). Parental contributions to externalizing and internalizing patterns in young children at risk for conduct disorder. Development and Psychopathology, 12(1), 23-45.

Derauf, C., LaGasse, L., Smith, L., Newman, E., Shah, E., Arria, A., et al. (2011). Infant temperament and high risk environment relate to behavior problems and language in toddlers. Journal of Developmental \& Behavioral Pediatrics, 32(2), 125-135.

Edgar, K. P., Schmidt L. A., Henderson, H. A., Schulkin, J., Fox, N. A. \& Feldman, R. (2008). Salivary cortisol levels and infant temperament shape developmental trajectories in boys at risk for behavioral Psychoneuroendocrinology 33(7), 916-925.

Eisenberg, N., Valiente, C., Spinrad, T.L., Cumberland, A., Liew, J.,Reiser, M., et al. (2009).Longitudinal relations of children's effortful control, impulsivity, and negative emotionality to their externalizing, internalizing, and co-occurring behavior problems. Developmental Psychology, 45(4), 988-1008.

Esser, G., Schmidt, M. H. \& Woerner, W. (1992). Epidemiology and course of psychiatric disorders in school-age children: Results of a longitudinal study. Journal of Child Psychology and Psychiatry, 31(2), 243-263.

Evans, D. E., \& Rothbart, M. K. (2007). Developing a model for adult temperament. Journal of Research in Personality, 41(4), 868-888.

Feldman, R. (2009). The development of regulatory functions from birth to 5 years: Insights from premature infants. Child Development, 80(2), 54461.

Flouri, E., \& Malmberg, L. E. (2010).Child temperament and paternal transition to nonresidence. Infant Behavior Development, 33(4), 689-694.

Gagne, J. R., \& Goldsmith, H. H. (2011). A longitudinal analysis of anger and inhibitory control in twins from 12 to 36 months of age. Developmental Science, 14(1), 112-124.

Garon, N., Bryson, S. E., Zwaigenbaum, L., Smith, I. M., Brian, J., Roberts, W., et al. (2009).Temperament and its relationship to autistic symptoms in a high-risk infant sib cohort. Journal of Abnormal Child Psychology, 37(1), 59-78.
Hanington, L., Ramchandani, P., \& Stein, A. (2010). Parental depression and child temperament: assessing child to parent effects in a longitudinal population study. Journal of Infant Behavior \& Development, 33(1), 88-95.

Hudson, J. L., Dodd H. F., Lyneham H. J. \& Bovopoulous, N. (2009) Temperament and family environment in the development of anxiety disorder: Two-year follow-up. Journal of the American Academy of Child and Adolescent Psychiatry, 50(12), 1255-1264.

Hwang, A. W., Soong, W. T., \& Liao, H. F. (2009). Influences of biological risk at birth and temperament on development at toddler and preschool ages. Child: Care, Health and Development, 35(6), 817-825.

Muris, P. \& Ollendick, T. H. (2005). The role of temperament in the etiology of child psychopathology. Clinical Child and Family Psychology Review, 8(4), 271-289.

Olson, S. I., Sameroff, A., Lunkenheimer, E. S., \& Kerr, D. (2009). Self-regulatory processes in the development of disruptive behavior problems: the pre-school to school transition. In: S.L. Olson \& A. J. Sameroff. Biopsychosocial regulatory processes in the development of childhood behavioral problems (pp. 144-185). New York: Cambridge University Press.

Perricone, G., \& Morales, M. R. (2011). The temperament of preterm infant in preschool age. Italian Journal of Pediatric, 37(4), 85-92.

Petitclerc, A., Boivin, M., Dionne, G., Zoccolillo, M., \& Tremblay, R. E. (2009). Disregard for rules: the early development and predictors of a specific dimension of disruptive behavior disorders. Journal of Child Psychology and Psychiatry, 50(12), 14771484.

Prior, M., Bavinb, E. L., Cinic, E., Reillyc, S., Brethertond, L., Wake, L. et al.(2008) Influences on communicative development at 24 months of age: Child temperament, behaviour problems, and maternal factors. Infant Behavior \& Development, 31(2), 270-279.

Rothbart, M. K. (1981). Measurement of temperament in infancy. Child Development, 52, 569-578.

Rothbart, M. K., Ahadi, S. A., \& Evans, D. E. (2000). Temperament and personality: Origins and outcomes. Journal of Personality and Social Psychology, 78(1), 122-135.

Rothbart, M. K. (2004). Commentary: Differentiated measures of temperament and multiple pathways to childhood disorders. Journal of Clinical Child and Adolescence Psychology, 33(1), 82-87.

Rothbart, M. K., \& Bates, J. E. (2006). Temperament. In: W. Damon, \& R. M. Lerner (Series Ed.), N. Eisenberg, (Vol. Ed.). Handbook of Child Psychology: social, emotional and personality development (pp. 99-165). New York: John Wiley and Sons.

Rothbart, M. K. \& Putnam, S. (2002).Temperament and socialization. In L. Pulkinnem \& A. Caspi 
(Orgs.), Paths to successful development: personality in the life course. Cambridge (pp. 1945). Cambridge University Press.

Sameroff, A. J. (2009). Conceptual issues in studying the development of self-regulation. In: S. L., Olson, \& A. J. Sameroff (Eds). Biopsychosocial regulatory processes in the development of childhood behavioral problems (pp. 1-18). New York: Cambridge University Press.

Stifter, C. A., Putnam, S., \& Jahromi, L (2008). Exuberant and inhibited toddlers: Stability of temperament and risk for problem behavior. Development and Psychopathology, 20(2), 401421.

Thomas, A., \& Chess, S. (1977). Temperament and Development. New York: Brunner/Mazel.

Vollrath, M. E., Tonstad, S., Rothbart, M. K., Hampson, S.E. (2011). Infant temperament is associated with potentially obesogenic diet at 18 months. International Journal Pediatric Obesity, 6(2-2), e408-e414.
Watamura S. E., Donzella, B., Kertes, D. A., \& Gunnar, M. R. (2004). Developmental changes in baseline cortisol activity in early childhood: relations with napping and effortful control. Developmental Psychobiology, 45(3), 125-133.

Whiteside-Mansell, L., Bradley, R. H., Casey, P. H., Fussell, J. J., \& Conners-Burrow, N. A. (2009). Triple risk: do difficult temperament and family conflict increase the likelihood of behavioral maladjustment in children born low birth weight and preterm? Journal of Pediatric Psychology, 34(4), 396-40.

Sofia Muniz Alves Gracioli: mestre pelo Programa em Saúde Mental da Faculdade de Medicina de Ribeirão Preto, Universidade de São Paulo.

Maria Beatriz Martins Linhares: professora associada do Departamento de Neurociências e Ciências do Comportamento da Faculdade de Medicina de Ribeirão Preto, Universidade de São Paulo. 